\title{
Il genere di SEO
}

\section{Anna M. Thornton}

PUBBLICATO: 12 FEBBRAIO 2021

\section{Quesito:}

Diverse persone chiedono quale sia il genere di SEO, acronimo di search engine optimization.

\section{Il genere di SEO}

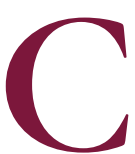

ome osservato già in alcune delle domande pervenute, nell'uso troviamo diffusi sia il SEO che la SEO.

Chi usa la SEO applica le due regole seguenti: I) gli acronimi ereditano il genere della loro testa; 2) i prestiti che vengono identificati con una corrispondente parola della lingua che li riceve prendono il genere di questa. Nel nostro caso, il nome inglese optimization viene identificato con il nome italiano ottimizzazione, e dunque riceve il genere femminile in base alla regola 2); poiché optimization è la testa del composto search engine optimization ('ottimizzazione per i motori di ricerca') che sta alla base dell'acronimo $S E O$, anche $S E O$ prende il genere femminile, in base alla regola i): la SEO.

Tuttavia, è possibile che qualcuno usi la parola SEO senza sapere sciogliere l'acronimo; può aver imparato questa forma e il suo significato come un tutto inanalizzabile. In tal caso, di fronte a una forma che termina in $o$, in italiano è normale assegnare il genere maschile. Ad esempio, al prestito kimono (dal giapponese, lingua priva della categoria di genere) è stato assegnato il maschile.

Il caso di acronimi usati con un genere diverso da quello della propria testa, per inconsapevolezza della sequenza esatta che è alla base dell'acronimo, non è raro. Per esempio, ONU, che abbrevia Organizzazione delle Nazioni Unite, dovrebbe essere femminile come organizzazione, ma è spesso usato al maschile. Nel corpus che raccoglie le annate $1985-2000$ del quotidiano "la Repubblica", consultabile online, troviamo entrambi gli usi: "L'Onu è stata a lungo unossessione della Casa Bianca", "l'Onu è diventata "la lunga mano di Mosca", ma anche "nel 50 per cento dei casi l'Onu è stato in grado di risolvere i problemi", "l'Onu è riuscito a imporre la pace".

Nello stesso corpus non abbiamo occorrenze di SEO, perché evidentemente questo termine tecnico si è diffuso in italiano dopo il 200o. Per verificarne l'uso ho quindi utilizzato il corpus Italian Web 2016 (itTenTen16). Si tratta di un corpus contenente testi scaricati da pagine web in italiano, contenente circa 5 miliardi di occorrenze di parole. In questo corpus ho esaminato le occorrenze di SEO preceduto dalle forme singolari dell'articolo determinativo il e la, anche in preposizione articolata; gli usi di SEO al femminile e al maschile sostanzialmente si bilanciano, con una lievissima prevalenza del femminile (I.924 vs. I.747, con un rapporto di I,I:I in favore del femminile).

Va però osservato che SEO è attestato in questo corpus anche in un altro senso, che si riferisce non all'attività di ottimizzazione, ma alle persone che svolgono questa attività, dette in inglese SEO specialists. Troviamo questo senso di SEO in esempi come i seguenti:

Ciao, mi presento: sono Giannicola Montesano e sono un SEO / SEM / Webmaster / Consulente Analista Programmatore; 
Sono un SEO e webmaster con oltre 5 anni d'esperienza;

Parlo per me: non sono un SEO, ma per i siti che sviluppiamo curo anche quell'aspetto.

In questo significato, come comunemente accade con i nomi d'agente che indicano attività professionali, abbiamo due possibilità: si usa il nome (che in questo caso è un acronimo e un prestito) con il genere congruente con quello della persona designata, oppure si usa un maschile generico per designare persone dei due sessi. Scegliendo la prima opzione Giannicola Montesano e altri si definiscono un SEO in quanto uomini, ma nello stesso corpus troviamo anche i testi seguenti, nei quali SEO è usato al femminile perché riferito a donne che svolgono questo lavoro:

Ciao sono Anna, ho zr anni e sono una SEO con specialistica nei Social Network;

Il blog di una SEO partita da Bari e finita a lavorare a Tel Aviv;

Jill Whalen, una SEO americana.

La seconda opzione si trova soprattutto al plurale, quando ci si riferisce in generale a persone che svolgono il lavoro di SEO, e che come abbiamo appena visto possono anche essere donne:

Su questo argomento molti SEO si stanno interrogando da qualche anno;

guadagnano molto, ma molto, ma molto più di molti SEO;

possiamo spiegarvi come lavorano molti SEO e come riescono a far precipitare il vostro sito dalle prime posizioni quando non pagate più il canone che vi chiedono.

In conclusione, $S E O^{1}$ nel senso di search engine optimization è preferibilmente femminile, come l'italiano ottimizzazione, ma è molto diffuso anche l'uso al maschile, favorito dalla opacità dell'acronimo e dal fatto che la parola termina in o; $S E O^{2}$ nel senso di 'specialista che lavora per ottenere una $S E O^{1}$ ' dovrebbe prendere il genere congruente con il sesso della persona designata.

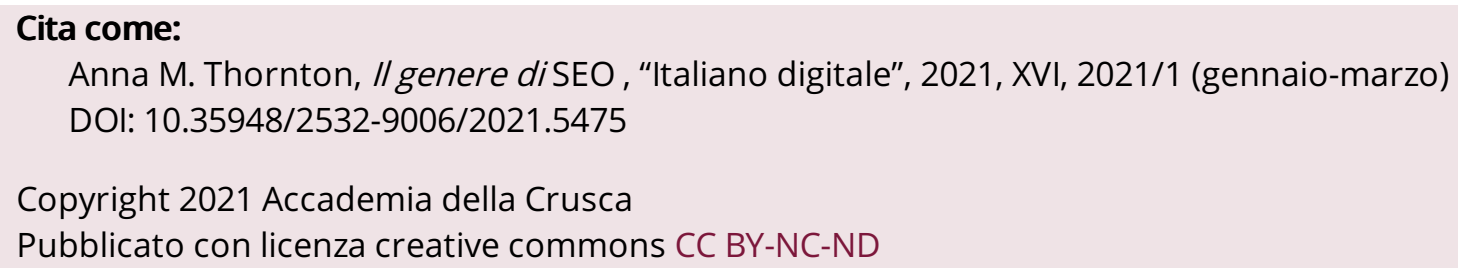

